
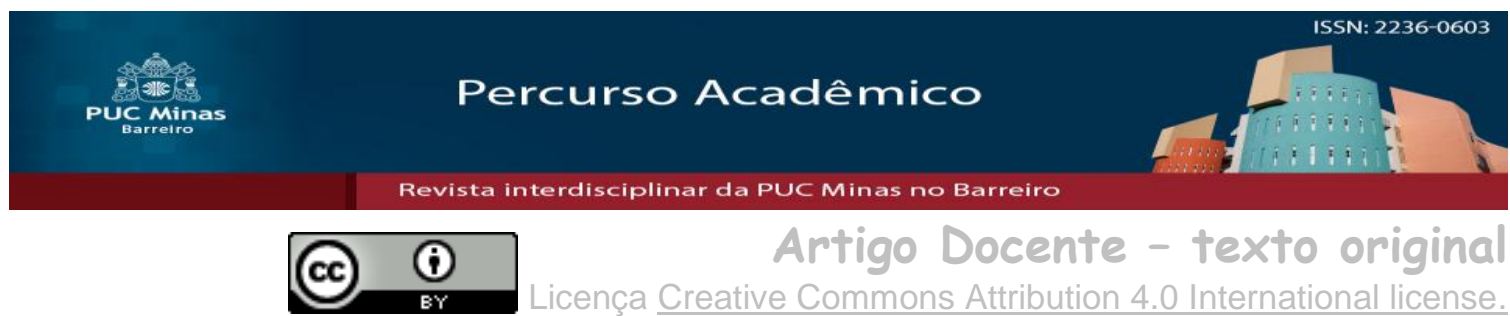

Licença Creative Commons Attribution 4.0 International license.

\title{
Apresentação de um Manual instrucional de promoção ao aleitamento materno em Unidade Básica de Saúde: um relato de experiência
}

\section{Presentation of a manual of instructions for promotion of breastfeeding at a Basic Health Unit: experience report.}

\author{
Amanda Rodrigues Garcia Palhoni ${ }^{1}$ \\ Bruna Figueiredo Manzo ${ }^{2}$ \\ Karine Antunes Marques Notaro ${ }^{3}$ \\ Lorena Lacerda Merlo Rocha ${ }^{4}$
}

\begin{abstract}
Resumo
O objetivo deste artigo é relatar a experiência da implementação de um Manual Instrucional de Promoção ao Aleitamento Materno aos profissionais das equipes de uma Unidade Básica de Saúde, de Belo Horizonte. Após a realização de um diagnóstico situacional, em relação a orientações dos profissionais às gestantes sobre o aleitamento materno, foi observado que as informações não eram sistematizadas e às vezes divergentes. Diante disso, foi elaborado o manual intitulado "Manual de Educação e Promoção ao Aleitamento Materno" conforme as recomendações estabelecidas pelo Ministério da Saúde, sendo proposto o uso do Kit Amamente da família Colchete ${ }^{\circledR}$, material didático composto por um boneco, mamas e um colete demonstrativo, como estratégia de treinamento e ferramenta de promoção ao aleitamento materno. Foi desenvolvida uma capacitação aos profissionais das equipes através de três encontros. Em cada encontro trabalhou-se uma temática específica relacionada à promoção do aleitamento materno, buscando garantir que práticas adequadas fossem instituídas, sendo os profissionais capacitados a oferecer suporte em aleitamento materno.
\end{abstract}

Palavras-chave: Aleitamento materno. Educação em saúde. Atenção Primária à Saúde.

\begin{abstract}
The purpose of this theme is to report the experience of implementing a Manual of Instructions to Promote Breastfeeding among the professional teams in the Basic Health Unit in Belo Horizonte. After accomplishing a situational diagnosis regarding guidelines of those professionals to pregnant women about breastfeeding, it has been observed that, informations were not systematized and sometimes they were divergent. Thus the manual was elaborated, entitled "Manual for Breastfeeding education and Promotion", according to the established recommendations the Ministry of Health, being proposed the use of the Kit called Breastfeed a family Colchet ${ }^{\circledR}$, a didactic material, which consists by a puppet with breast and a showing vest as a strategy and training tool to promote the breastfeeding. A training course has been developed for professionals teams through three meetings. Each meeting has worked out a specific theme related to breastfeeding promotions, in order to ensure that appropriate breastfeeding practices be instituted and the professionals able to support breastfeeding practices.
\end{abstract}

Keywords: Breastfeeding. Health education. Primary Health Care.

\footnotetext{
Artigo recebido em 05 de agosto de 2014 e aprovado em 22 de fevereiro de 2016.

${ }^{1}$ Mestre em Enfermagem. Especialista em Enfermagem Obstétrica pela UFMG. Professora Substituta do Departamento Materno Infantil e Saúde Pública da Escola de Enfermagem da UFMG. E-mail: amandargpalhoni@yahoo.com.br

${ }^{2}$ Doutora em Enfermagem. Professora Adjunta do Departamento de Enfermagem materno infantil e saúde publica da Universidade federal de Minas Gerais. Belo Horizonte, Minas Gerais, Brasil. E-mail: brunaamancio@yahoo.com.br

${ }^{3}$ Enfermeira. Especialista em Enfermagem Hospitalar Clínico-Cirúrgico e em Acreditação: Qualidade no Serviço de Saúde. Enfermeira do Banco de Leite da Maternidade Odete Valadares. E-mail: karineantuneshob@yahoo.com.br

${ }^{4}$ Enfermeira. Especialista em Terapia Intensiva Neonatal e Pediátrica. Enfermeira da Neonatologia da Maternidade Odete Valadares. Email: lorenalmerlo@hotmail.com
} 


\section{Introdução}

A importância do aleitamento materno foi consagrada no século $\mathrm{XX}$, representando a estratégia de maior impacto na diminuição da mortalidade infantil. (BARBOSA; MACHADO, 2010). Amamentar, além de nutrir, promove o vínculo afetivo entre mãe e filho e tem repercussões na habilidade da criança de se defender de infecções, em sua fisiologia e em seu desenvolvimento cognitivo e emocional, assim como na saúde física e psíquica da mãe. (BRASIL, 2011).

A Organização Mundial de Saúde (OMS) e o Fundo das Nações Unidas para a Infância e Adolescência (UNICEF) recomendam que todos os bebês sejam amamentados com leite materno, exclusivamente, até o sexto mês de vida; depois disso o aleitamento materno deve ser complementado com outros alimentos até dois anos ou mais, recomendação esta também adotada oficialmente no Brasil. (UNICEF, 2009).

Apesar do aumento das taxas de amamentação na maioria dos países nas últimas décadas, inclusive no Brasil, a tendência ao desmame precoce continua, e o número de crianças amamentadas segundo as recomendações da Organização Mundial da Saúde (OMS) ainda é pequeno. (BRASIL, 2010).

Em todo o mundo, apenas $35 \%$ de crianças recebem aleitamento materno exclusivo durante os primeiros quatro meses de vida. No Brasil, dados coletados na última Pesquisa Nacional de Demografia e Saúde da Criança e da Mulher - PNDS-2006 demonstraram que a duração mediana de aleitamento materno exclusivo no Brasil, foi de 1.4 meses. (BRASIL, 2009). Em Belo Horizonte (BH), Minas Gerais, segundo a Pesquisa de Prevalência de Aleitamento Materno em Municípios Brasileiros em crianças menores de seis meses em amamentação exclusiva, corresponde a 37,9\%. (BRASIL, 2010).

Políticas Públicas de Saúde têm sido lançadas como esforços no sentido de incentivar a prática de amamentação, obtendo-se graus variados de sucesso. (REZENDE et al, 2002). Iniciativas como o Programa Nacional de Incentivo ao Aleitamento Materno, Hospital Amigo da Criança, Rede Nacional de Bancos de Leite Humano, a prática do Alojamento Conjunto, Bombeiros Amigos da Amamentação e Carteiro Amigo, Método Mãe Canguru, Semana Mundial da Amamentação são ações criadas pelo governo com o enfoque na promoção do aleitamento materno a fim de reduzir a mortalidade neonatal e infantil. (BRASIL, 2010; SAKAE; COSTA; VAZ, 2001). 
Na Atenção Básica (AB), a Estratégia Saúde da Família (ESF) vem se consolidando como um dos eixos estruturantes do Sistema Único de Saúde (SUS), por meio de um movimento de expressiva expansão de cobertura populacional, aprimorando em muito o acesso da população às ações de saúde. Dentro desse processo, o Pacto pela Redução da Mortalidade Materna e Neonatal, o Pacto pela Vida e a Política Nacional de Atenção Básica vieram para contribuir como instrumentos para o fortalecimento da saúde da família no âmbito do SUS. (BRASIL, 2009).

A ESF tem relevante importância na prática do aleitamento, as orientações acontecem desde o planejamento familiar, continuando no pré-natal e se estendendo do puerpério até a fase de aleitamento materno exclusivo na puericultura. (ARAUJO et al., 2013). Entretanto, é um desafio para os profissionais da saúde garantirem que práticas adequadas em aleitamento materno sejam instituídas, oferecendo suporte em aleitamento materno a todos que necessitam, respeitando os valores sociais, culturais e as mudanças da estrutura familiar na sociedade moderna.

As dificuldades encontradas pela mulher no momento de amamentar são enfatizadas por diversos autores (REZENDE et al., 2002; ALMEIDA; NOVAK, 2004; CARVALHAES; CORREA, 2003;) e o desmame precoce ainda é predominante. Batista; Farias; Melo (2013) supõem que uma das justificativas para essa realidade seja o fato de os profissionais de saúde não estarem próximos à mulher no processo de lactação. O profissional de Enfermagem deve ser capaz de identificar as dificuldades e atuar na promoção do aleitamento materno.

Souza; Mello; Ayres (2013) demonstraram em seu estudo que a maneira como as mães e famílias lidam com o aleitamento materno está ligada ao modo como as práticas estão organizadas e com aspectos individuais e sociais, daí a necessidade de integração entre saberes práticos e técnico-científicos.

Estudo sobre o impacto da atualização entre profissionais de saúde da $\mathrm{AB}$, em Porto Alegre, em relação aos Dez Passos da Alimentação Saudável para Crianças Menores de Dois Anos, sobre as práticas alimentares no primeiro ano de vida, demonstrou que a atualização de profissionais pode ser uma estratégia para aumentar a prevalência de crianças amamentadas exclusivamente nos primeiros seis meses de vida. A interrupção precoce do aleitamento materno exclusivo, nos primeiros seis meses, foi menor entre as crianças atendidas em dois serviços em que os profissionais receberam a atualização sobre o tema. (VÍTOLO et al., 2014). A educação e o preparo das mulheres para lactação, durante o período do pré-natal, 
comprovadamente contribui para o sucesso do aleitamento materno. (BATISTA; FARIAS; MELO, 2013).

Durante a participação nas atividades diárias do enfermeiro, inclusive no pré-natal, e também com os demais integrantes da equipe, foi notado pelos discentes, durante o estágio supervisionado em uma Unidade Básica de Saúde (UBS) em BH, que as orientações às gestantes em relação à amamentação não eram sistematizadas e até mesmo divergentes. Surge então a ideia de capacitação desses profissionais por meio da elaboração prévia de um Manual Instrucional de Promoção ao Aleitamento Materno que pudesse ser utilizado por toda(s) a(s) equipe(s), norteando-a(s) no processo de promoção e preparo das mulheres nas práticas relacionadas ao aleitamento materno. Esta ideia foi compartilhada junto com a enfermeira da UBS.

Acredita-se ser fundamental propor estratégias centradas no aspecto educativo, abrangendo tanto a difusão de informações quanto a capacitação dos profissionais que estão diretamente ligados à mulher durante seu ciclo gravídico-puerperal e, em especial, durante a realização do pré-natal, sendo uma prática recomendada pelo Ministério da Saúde (MS).

Este estudo tem como objetivo relatar a experiência da implementação de um Manual Instrucional de Promoção ao Aleitamento Materno, proposto por discentes de Enfermagem, por meio de uma capacitação aos profissionais das equipes de saúde (técnicos, enfermeiros, auxiliares de Enfermagem e agentes comunitários de saúde) de uma UBS de BH.

\section{Metodologia}

\subsection{O Cenário}

O trabalho foi desenvolvido em uma UBS da região norte de BH. Esta foi inaugurada em 1995, e deste então realiza atendimento a todos os ciclos de vida. Em 2000 foi implantado o Programa de Agentes Comunitários (PAC's) e em 2002 oficialmente instituiu-se o Programa Saúde da Família, atualmente definido como ESF, com a criação da primeira equipe de saúde da família.

Atualmente a UBS é constituída por três equipes de saúde da família, que abrange uma população total adscrita de 14.004 pessoas, distribuídas em doze micro áreas, em sua maioria classificada como elevado risco de vulnerabilidade social.

As gestantes da área de abrangência são acompanhadas durante o período do pré-natal através de consultas intercaladas por profissionais médicos generalistas e/ou ginecologista e 
enfermeiros. Os grupos de gestantes não são realizados de forma periódica e as orientações relacionadas à amamentação e ao parto são realizadas somente de forma individual.

Durante a gestação, o Agente Comunitário de Saúde (ACS) realiza a visita domiciliar buscando acompanhar o estado de saúde e intercorrências, após o parto a puérpera é geralmente visitada em seu domicílio pelo enfermeiro e ACS, no quinto dia de vida do recémnascido $(\mathrm{RN})$.

\subsection{O Projeto}

Após extensa revisão literária e observação da realidade local durante o estágio supervisionado, elaboramos o manual intitulado "Manual de Educação e Promoção ao Aleitamento Materno", conforme as recomendações estabelecidas pelo MS. A ferramenta de promoção ao aleitamento proposta é composta pelo manual e pelo Kit Amamente da família Colchete $^{\circledR}$, material didático conhecido durante as aulas de Saúde da Mulher e do RN e que é composto por um boneco, quatro mamas enumeradas de um a três e um colete demonstrativo.

Após a elaboração do manual, a capacitação foi agendada e realizada nas dependências da UBS, aonde vinha acontecendo o estágio supervisionado no ano de 2012. Contou com a participação de três discentes, um docente e oito profissionais de saúde.

Foram desenvolvidas técnicas de oficina com a utilização recursos visuais e materiais que despertassem o interesse em discutir o tema, levantar dúvidas e possibilitar espaço para trocas experiências. Segundo Souza et al. (2012) a proposta da oficina apresenta similaridade com a visão filosófica, política e metodológica da educação defendida por Paulo Freire, que baseia-se na experiência vivencial e no desenvolvimento da consciência crítica dos participantes. Elas não seguem norma rígida, podendo ser construída a cada sessão, tendo em vista os objetivos do trabalho e as demandas identificadas pelo grupo.

Entretanto, para Souza et al. (2012) algumas etapas fundamentais devem ser observadas tais como: o desenvolvimento um planejamento flexível e que leve em conta as demandas dos participantes, a definição de um tema central, disponibilização de um local confortável e que tenha consonância com as atividades planejadas, entre outros.

\section{Relatando a experiência e discutindo os resultados}

Os encontros com os profissionais da UBS foram autorizados previamente pela gerente da unidade e viabilizados com o apoio da enfermeira, referência nesta área de atendimento de saúde da mulher e do RN. Ocorreram em três encontros como pode se notar 
no Quadro 1 e foram em dias consecutivos, três vezes por semana. Em cada encontro trabalhou-se uma temática específica relacionada à promoção do aleitamento materno sendo dividido em quatro momentos, com duração média de duas horas e trinta minutos.

Quadro 1: Esquema proposto para a capacitação dos profissionais Belo Horizonte

\begin{tabular}{|l|l|}
\hline Atividades Educativas & \multicolumn{1}{|c|}{ Tema } \\
\hline Encontro 1 & $\begin{array}{l}\text { "Entendendo a anatomia da mama e as vantagens do } \\
\text { aleitamento materno" }\end{array}$ \\
\hline Encontro 2 & "Aprendendo a amamentar" \\
\hline Encontro 3 & "Evitando as complicações e como fazer doação de leite” \\
\hline
\end{tabular}

Fonte: Elaborado pelos autores, 2012.

A seguir estratégias utilizadas nos encontros foram descritas, relatando o decorrer de cada dia e a avaliação do instrumento proposto.

- $1^{\circ}$ encontro: "Entendendo a anatomia da mama e as vantagens do aleitamento materno".

Foram capacitados cinco técnicos de Enfermagem, três ACS's e três acadêmicas de Enfermagem. Os temas abordados foram: "Anatomia da mama", "Vantagens e importância do aleitamento materno" e "Preparo das mamas durante a gestação".

$1^{o}$ momento: Apresentação dos discentes e participantes. Além da apresentação da proposta de trabalho, procurou-se conhecer os integrantes do grupo por meio de uma dinâmica intitulada "Dinâmica do Presente", com objetivo de trabalhar a atenção, a observação, o desapego, a sinceridade. Nessa dinâmica, cada participante é estimulado a dar e repartir um objeto que traz boas lembranças, estabelecendo empatia entre os participantes. Foi um momento de descontração que proporcionou aproximação entre os participantes e entre participantes e discentes.

$2^{o}$ momento: Troca de experiências. A fim de identificar a demanda de capacitação pelos discentes, o grupo foi estimulado a relatar dúvidas, experiências e conhecimentos prévios sobre o tema. As principais dúvidas que surgiram foram relacionadas à qualidade do leite materno, posicionamento da criança, complementação com leite de fórmula. Entendemos que começar a oficina a partir do aproveitamento do conhecimento e questionamentos dos participantes foi uma estratégia importante, uma vez, que valoriza o que as pessoas sabem 
sobre o assunto e permite que se desenvolva um trabalho de acordo com as necessidades dos sujeitos envolvidos.

$3^{o}$ momento: Discussão do tema proposto. Iniciamos a demonstração com a mama do kit Amamente®, que se assemelha muito às características da mama humana no tamanho, forma etc. O objetivo dessa introdução foi o esclarecimento do grupo acerca do conhecimento sobre a anatomia e fisiologia da mama, aspectos estes que podem interferir no processo de amamentação.

$4^{o}$ momento: Avaliação da atividade. Para finalização do primeiro encontro foi realizada uma avaliação como forma de verificar o entendimento do conteúdo discutido. Para tal utilizamos uma dinâmica de perguntas e respostas, previamente elaboradas, embasadas no conteúdo didático proposto para aquele dia e outras baseadas nas dúvidas iniciais das participantes.

Todos participantes interagiram com o material proposto e demonstraram entendimento, respondendo adequadamente às perguntas propostas.

- $2^{\circ}$ encontro: "Aprendendo a amamentar"

Houve a participação de seis técnicos, três ACS's e três acadêmicas de Enfermagem. Foram abordados os seguintes temas: "Colostro, apojadura e oferta do leite ao recémnascido", "Técnica correta de amamentar" e "Posicionamento do bebê e da mãe".

$1^{o}$ momento: Chegada e descontração. Foi promovida a interação entre os participantes por meio de um café da manhã de boas vindas e uma breve revisão dos assuntos abordados no primeiro encontro sobre anatomia das mamas e vantagens do aleitamento.

$2^{o}$ momento: Sondagem. A sondagem é uma verificação que se segue de constatação, é avaliar e estimar o conhecimento prévio que os participantes possam ter a respeito do assunto. Reconhecemos erros e acertos acerca do questionamento dos profissionais em relação ao tema a ser abordado no segundo encontro sobre colostro, apojadura e oferta de leite ao recémnascido, que norteou o desenvolvimento do próximo momento. A avaliação foi feita a partir de perguntas orais aos participantes, perguntas estas que seriam esclarecidas e abordadas ao longo da capacitação.

$3^{o}$ momento: Exposição do tema proposto. Os temas foram abordados através de recursos visuais como cartazes de cartolina com imagens e textos explicativos sobre o tema e placas confeccionadas pelas próprias discentes contendo imagens disponíveis no Manual Instrucional, mostrando a pega e posição do bebê correta do bebê ao seio. 
$4^{o}$ momento: Avaliação. Foram utilizados o boneco e as mamas que compõem o kit Amamente $^{\circledR}$ a fim de verificar a compreensão do grupo acerca dos temas abordados. Todos os participantes demonstraram clareza no entendimento da técnica de amamentação e do posicionamento mãe-bebê.

- $3^{\circ}$ encontro: "Evitando as complicações e como fazer doação de leite"

Participaram do grupo seis técnicos, três ACS's e três acadêmicas de Enfermagem. Foram abordados os seguintes temas: "Como evitar as complicações relacionadas ao aleitamento materno", "Ordenha da mama", "Armazenamento e conservação do leite" e "Banco de Leite Humano".

$1^{o}$ momento: Chegada e sondagem. Após a revisão dos assuntos abordados no segundo encontro sobre técnicas corretas de amamentação, iniciamos a sondagem e, para tanto, desenvolveu-se uma atividade de verificação das opiniões dos profissionais acerca de experiências negativas durante o processo de amamentar. Alguns profissionais relataram experiências próprias que dificultaram a amamentação e as fizeram não dar continuidade tais como: ter bico do peito plano, pelo fato da mãe ter dito que seu leite era fraco e o bebê chorar de fome, pelo fato do mamilo ferir e não saber o que fazer.

$2^{o}$ momento: Exposição do tema proposto. Informações importantes. Utilizando técnicas de auxílio visual e as mamas do kit Amamente ${ }^{\circledR}$, desenvolvemos o tema relacionado às possíveis complicações no aleitamento materno através da comparação de dois lados da mama: lado A (mama empedrada) e B (mama normal).

A ordenha da mama foi demonstrada com a utilização do colete e das mamas do kit. Os participantes foram então sensibilizados quanto à importância da doação de leite humano e informados quanto ao Banco de Leite Humano de referência e suas funções.

$3^{o}$ momento: Avaliação. O grupo foi estimulado a identificar as possíveis complicações em relação ao aleitamento materno e propor intervenções por meio do kit, e responder perguntas previamente estabelecidas. Demonstraram habilidade e compreensão dos temas e técnicas abordados. De todos os encontros, esse terceiro foi o que mais surgiu dúvidas e que, de certa forma, reafirmou a importância da capacitação para esses profissionais na promoção do aleitamento materno.

$4^{o}$ momento: Distribuição das cartilhas. Como eixo norteador do trabalho de promoção ao aleitamento materno, foi elaborado uma cartilha intitulada: "Aleitamento Materno, Alimento e Amor" anexa ao manual, contendo informações objetivas e de fácil entendimento 
dos temas desenvolvidos. Esta foi apresentada ao grupo e estimulada a ser fornecida pelos profissionais às gestantes, ao final do terceiro encontro, quando os mesmos forem reproduzir essas oficinas com as gestantes, como um instrumento de informações e de consulta rápida em caso de dúvidas. A cartilha foi construída durante a realização dos encontros, levando em consideração as ações desenvolvidas anteriormente, destacando e respondendo as dúvidas apontadas pelos participantes e embasada na literatura.

Neste último encontro foi realizada uma avaliação envolvendo os três encontros, utilizando, para tal, um questionário avaliativo baseado em uma escala de satisfação por expressão facial (ótimo, bom e regular). As questões envolviam perguntas como: O manual é viável para ser usado na UBS? O manual engloba todos os assuntos relacionados ao aleitamento materno? O manual colaborou ou esclareceu dúvidas em relação à amamentação? Sobre a satisfação em relação ao manual e sobre o kit didático apresentado, foi pedido para que marcassem a resposta que mais se relacionava com a opinião de cada um, tendo como opções: ótimo, bom, regular e ruim, através de expressões faciais. Todos os participantes verbalizaram estar muito satisfeitos com o manual proposto, além disso, todos marcaram como resposta a expressão facial correspondente a ótimo.

Houve sugestões verbais como o desenvolvimento da capacitação com periodicidade maior, a possibilidade de uma maior participação dos outros profissionais e a inclusão dos profissionais médicos. Estudo realizado com médicos, nos Estados Unidos, corroboram as sugestões acima, pois mostrou que, apesar destes reconhecerem seu potencial em influenciar positivamente o aleitamento materno, relatavam limitada capacitação específica para promoverem a prática. (KROGSTRAND; PARR, 2005).

A capacitação proporcionou reflexões, troca de experiências e ampliação do conhecimento acerca da promoção e incentivo a amamentação. Brasil (2011) enfatiza que a promoção da saúde é o processo que vai possibilitar a cada população avaliar a qualidade de vida que lhe é oferecida e atuar sobre ela para conseguir cada vez mais saúde.

Esta avaliação demonstra o resultado positivo da capacitação desenvolvida. Houve um comprometimento da equipe em dar continuidade no uso do manual proposto, entretanto a prática do aleitamento materno há de se considerar não somente os aspectos fisiológicos da amamentação, mas também os aspectos emocionais e sociais da gestante e puérpera.

Neste processo, cabe ao profissional da saúde contribuir para a orientação e apoio às pacientes. (BATISTA; FARIAS; MELO, 2013). Conforme Percegoni et al (2002), existe um 
baixo nível de conhecimento, por parte das puérperas, sobre questões fundamentais para o sucesso da amamentação reafirmando a importância do incentivo e da orientação para o aleitamento durante o pré-natal.

Sendo o pré-natal uma ótima oportunidade para orientar e incentivar às mães a amamentarem seus filhos, Vasconcelos; Lira; Lima (2006) corroboram essa perspectiva no estudo apresentado, no qual ocorreu uma relação diretamente proporcional entre o número de consultas realizadas no pré-natal e o aumento da duração mediana do aleitamento total.

Mesmo comprovadamente eficaz, a orientação para amamentação durante o pré-natal não é realizada corretamente pelos profissionais, conforme relatado por Rios; Vieira (2007), que perceberam, através das falas das participantes, a necessidade de se gerar esforços para a realização da assistência educativa como forma de melhorar o impacto dessa ação na saúde física, mental e emocional da gestante durante o pré-natal, quer individualmente ou mesmo coletivamente.

Caldeira et al (2007), em seus resultados, revelam que é possível melhorar o desempenho dos profissionais da ESF em relação à amamentação, o que determina o compromisso social de sensibilizar e capacitar toda a equipe.

Na realização do momento "Troca de experiências", a fimm de identificar a demanda, o grupo foi estimulado a relatar dúvidas, experiências e conhecimentos prévios sobre o tema, o que permitiu a construção do conhecimento do profissional a partir da reflexão sobre o que se sabe e a absorção de novos conhecimentos com o relato e troca de experiências com os colegas. O desenvolvimento de trabalhos em grupo é essencial, pois proporciona uma maior aproximação dos aspectos teóricos que foram construídos com o profissional e a prática realizada na assistência.

\section{Considerações Finais}

No decorrer de todo o processo de elaboração da capacitação, o cuidado na escolha e desenvolvimento das atividades propostas, em cada um dos encontros, foi marcado pelo objetivo de promover a interação e a empatia entre os participantes, de modo a incentivar e sensibilizar a promoção do aleitamento materno.

Destacou-se, portanto, para fins da realização desses encontros, a importância da capacitação desenvolvida, na medida em que é viável para utilização posterior na UBS em questão, tanto para outros profissionais como para usuárias do serviço. Nesse sentido, contribui-se para padronizar os processos envolvidos na promoção do aleitamento materno, de 
forma a cooperar para a diminuição de práticas divergentes e atuar como eixo orientador às práticas propostas. Interessante ressaltar que seria necessário envolver profissionais médicos na atividade, pois as dúvidas mais frequentes desses profissionais voltavam-se para manejo dos principais problemas de amamentação. Acreditamos que é preciso diminuir a distância que existe, não somente em relação ao aleitamento materno, no caso desse relato de experiência, mas também na área da saúde de modo geral, do que acontece na prática em relação ao conhecimento científico. Para isso, torna-se importante desenvolvimento de pesquisas que possam ser aplicadas no contexto prático em relação ao aleitamento materno e de educação continuada para esses profissionais.

Ressaltamos ser fundamental propor estratégias centradas no aspecto educativo, abrangendo tanto a difusão de informações quanto a capacitação dos profissionais inseridos na $\mathrm{AB}$, e que estão diretamente ligados à mulher durante o seu ciclo gravídico-puerperal para promoção do aleitamento materno e prevenção do desmame precoce.

\section{REFERÊNCIAS}

ALMEIDA, J.A.G.; NOVAK, F.R. Amamentação: um híbrido natureza-cultura. J. Pediatr. Rio Janeiro, v. 80, n. 5, nov. 2004. Disponível em: http://www.scielo.br/scielo. Acesso em: 03 agosto de 2014 .

ARAÚJO J.P.; ALMEIDA J.L.S.; SOUTO C.M.R.M.; OLIVEIRA A.E.A., SUDÉRIO M.A.R.P. Desmame precoce e suas causas: experiência na atenção básica de campina grandepb. Revista da Universidade Vale do Rio Verde, Três Corações, v. 11, n. 2, p. 146-155, ago./dez. 2013.

BARBOSA N.B.S.; Machado M.M. Aleitamento materno no município de Anápolis: saberes e práticas na estratégia saúde da família. Revista APS, Goiás, v. 13, n. 4, 2010.

BATISTA K.R.A.; FARIAS M.C.A.D.; MELO W.S.N. Influência da assistência de enfermagem na prática da amamentação no puerpério imediato. Saúde em Debate, Rio de Janeiro, v. 37, n. 96, p. 130-138, jan./mar. 2013.

BRASIL. Ministério da Saúde. Pesquisa de Prevalência de Aleitamento Materno em Municípios Brasileiros. Situação do Aleitamento Materno em 227 municípios brasileiros Brasília - DF, 2010. Disponível em: http://www.redeblh.fiocruz.br/media/pamuni.pdf. Acesso em: 07 maio de 2014.

BRASIL. Ministério da Saúde. Secretaria de Atenção à Saúde. Departamento de Ações Programáticas e Estratégicas. Atenção à Saúde do Recém-Nascido: guia prático para profissionais de saúde. Brasília: Editora do Ministério da Saúde, v.1, p.192, 2011. 
BRASIL. Ministério da Saúde. Secretaria de Atenção à Saúde. Departamento de Atenção Básica. Saúde da criança: nutrição infantil: aleitamento materno e alimentação complementar / Ministério da Saúde, Secretaria de Atenção à Saúde, Departamento de Atenção Básica. Brasília: Ministério da Saúde, 2009.112 p.: il. - (Série A. Normas e Manuais Técnicos) (Cadernos de Atenção Básica, n. 23).

CALDEIRA A.P.; AGUIAR G.N., MAGALHÃES W.A.C., FAGUNDES G.C. Conhecimentos e práticas de promoção do aleitamento materno em Equipes de Saúde da Família em Montes Claros, Minas Gerais, Brasil. Cad. Saúde Pública, Rio de Janeiro, v. 23, n.8, p. 1965-1970, 2007.

CARVALHAES M.A.B.L.; CORREA, C.R.H. Identificação de dificuldades no início do aleitamento materno mediante aplicação de protocolo. J. Pediatr., Rio de Janeiro, v.79, p.1320, 2003. Disponível em: http://www.scielo.br/scielo. Acesso em: 25 fev. 2013.

KROGSTRAND K.S.; PARR, K. Physicians ask for more problem-solving information to promote and support breastfeeding. J. Am. Diet Assoc.; Estados Unidos da América, v. 105, p. 1943-1947, 2005.

PERCEGONI, Nathércia et al . Conhecimento sobre aleitamento materno de puérperas atendidas em dois hospitais de Viçosa, Minas Gerais. Rev. Nutr., Campinas , v. 15, n. 1, p. 29-35, Jan. 2002. Available from:

$<$ http://www.scielo.br/scielo.php?script=sci_arttext\&pid=S1415$52732002000100004 \& \operatorname{lng}=$ en\&nrm=iso $>$. access on 13 Sept. 2015. http://dx.doi.org/10.1590/S1415-52732002000100004.

REZENDE M.A.; SIGAUD, C.H.S., VERÍSSIMO, MDeLaÓR; CHIESA, A.M., BERTOLOZZI, M.R. O processo de comunicação na promoção do aleitamento materno. Rev. Latino-Am. Enfermagem, Ribeirão Preto- SP, v. 10, n. 2, p. 234-238, mar./abr. 2002. Disponivel em: http://dx.doi.org/10. 1590/S0104-11692002000200017 Acesso em: 02 abril 2008.

RIOS, Claudia Teresa Frias; VIEIRA, Neiva Francenely Cunha. Ações educativas no prénatal: reflexão sobre a consulta de enfermagem como um espaço para educação em saúde. Ciênc. saúde coletiva, Rio de Janeiro, v. 12, n. 2, p. 477-486, abr. 2007. Disponível em $<$ http://www.scielosp.org/scielo.php?script=sci_arttext\&pid=S1413$81232007000200024 \& \operatorname{lng}=$ pt\&nrm=iso $>$. acesso em 13 set. 2015. http://dx.doi.org/10.1590/S1413-81232007000200024.

SAKAE P.P.O.; COSTA, M.T.Z., VAZ, F.A.C. Cuidados perinatais humanizados e o aleitamento materno promovendo a redução da mortalidade infantil. Pediatria, São Paulo, v. 23, n. 2, p. 179-187. 2001. Disponível em: http://www.scielo.br/scielo. Acesso em 25 fevereiro de 2008.

SOUZA, K. V. et al. Conhecendo o Corpo que se Altera na Gestação. In: FIGUEIREDO, N. M. A; MACHADO, W. C. A. (Org.). Tratado de Cuidados de Enfermagem MédicoCirúrgico. São Paulo: Roca, 2012. Volume II, Capítulo 101, p. 1672-1687. 
SOUZA, S.N.D.H.; MELLO, D.F.; AYRES, J.R.C.M. O aleitamento materno na perspectiva da vulnerabilidade programática e do cuidado. Cad. Saúde Pública, Rio de Janeiro, v. 29, n. 6, jun. 2013.

\section{UNICEF. The Baby-Friendly Hospital Initiative. Word Health Organization and}

UNICEF. 2009. Disponível em:

http://www.who.int/nutrition/publications/infantfeeding/bfhi_trainingcourse/en/ Acesso em 10 de setembro de 2015.

VASCONCELOS, Maria Gorete Lucena de; LIRA, Pedro Israel Cabral de; LIMA, Marília de Carvalho. Duração e fatores associados ao aleitamento materno em crianças menores de 24 meses de idade no estado de Pernambuco. Rev. Bras. Saúde Mater. Infant., Recife , v. 6, n. 1, p. 99-105, Mar. 2006. Disponível em:

$<$ http://www.scielo.br/scielo.php?script=sci_arttext\&pid=S1519-

38292006000100012\&lng=en\&nrm=iso> Acesso em 13 Sept 2015.

http://dx.doi.org/10.1590/S1519-38292006000100012.

VITOLO M.R. et al. Impacto da atualização de profissionais de saúde sobre as práticas de amamentação e alimentação complementar. Cad. Saúde Pública, Rio de Janeiro, v. 30, n. 8, p. 1695-1707, ago. 2014. 